BESSIRE Lucas, Behold the black caiman. A chronicle of Ayoreo life

The University of Chicago Press, Chicago/Londres, 2014

\title{
Alfonso Otaegui
}

\section{(2) OpenEdition}

\section{Journals}

Edición electrónica

URL: https://journals.openedition.org/jsa/14212

DOI: $10.4000 /$ jsa. 14212

ISSN: 1957-7842

\section{Editor}

Société des américanistes

\section{Edición impresa}

Fecha de publicación: 31 diciembre 2015

Paginación: 332-339

ISSN: 0037-9174

\section{Referencia electrónica}

Alfonso Otaegui, «BEssine Lucas, Behold the black caiman. A chronicle of Ayoreo life», Journal de la Société des américanistes [En línea], 101-1 et 2 | 2015, Publicado el 15 marzo 2016, consultado el 24 febrero 2023. URL: http://journals.openedition.org/jsa/14212 ; DOI: https://doi.org/10.4000/jsa. 14212

Este documento fue generado automáticamente el 24 febrero 2023

All rights reserved 


\title{
BESSIRE Lucas, Behold the black caiman. A chronicle of Ayoreo life
}

The University of Chicago Press, Chicago/Londres, 2014

\author{
Alfonso Otaegui
}

\section{REFERENCIA}

BESSIRE Lucas, Behold the black caiman. A chronicle of Ayoreo life, The University of Chicago Press, Chicago/Londres, 2014, 314 p., bibliogr., index, ill., photos.

1 En su libro Behold the black caiman. A chronicle of Ayoreo life (que podría traducirse como Contemplad el caimán negro. Una crónica de vida ayoreo), Lucas Bessire sigue de cerca el destino de un pequeño grupo de ayoreo totobiegosode que salió del monte en 2004 y el de otros ayoreo ya contactados décadas antes. El autor se esfuerza desde el comienzo por distanciarse de las dinámicas interpretativas habituales que hablarían del contacto como degradación, como pérdida de una tradición hasta entonces inalterada. En vez de ello, Bessire propone estudiar el devenir de los ayoreo, y se aboca a defender la capacidad de auto-objetivación de este grupo. El libro abarca una amplia variedad de temas, desde el análisis de mitos y fórmulas de cura, el uso de nuevas tecnologías, la conversión al cristianismo, hasta el rol que misioneros, antropólogos y ONG han jugado en la vida e historia de este grupo del Chaco boreal.

2 En cuanto a su estilo narrativo, el libro está muy bien escrito y su lectura es llevadera. Las numerosas anécdotas - instiladas generalmente al comienzo y a final de cada capítulo - ilustran las problemáticas discutidas y le dan una textura de "experiencia vivida " a la investigación. Bessire nos permite de ese modo percibir, como en una crónica, las vidas de los ayoreo en el Chaco paraguayo y en Bolivia.

Si bien la variedad de temas abarcados es amplia, hay una serie de ideas rectoras que atraviesan toda la obra. Bessire postula aquí fundamentalmente la necesidad de abandonar el estudio de una alteridad radical indígena - espacio de la nomodernidad -, y concentrarse en el devenir, en el «llegar a ser » ("becoming») de los 
ayoreo, reconociéndoles su capacidad para la autodeterminación ontológica. El autor discutirá a lo largo de toda la obra con la figura del antropólogo primitivista que va al campo a registrar costumbres del pasado, y que está ciego frente a la realidad presente. También irá contra el "giro ontológico"-en particular el perspectivismo-, y analizará las consecuencias políticas que - a juicio del autor - esa propuesta acarrea. Bessire analiza incisivamente el papel que juegan las ONG en el campo ayoreo, sus estrategias focalizadas en los grupos aislados y las consecuencias nocivas que esas políticas tienen para los « ex-primitivos », carentes de suficiente alteridad.

El autor construye la figura alegórica del caimán negro como una imagen de incertidumbre, violencia y muerte que en cierto modo representa las ambigüedades del ser ayoreo en el mundo actual. Podría decirse que el siguiente párrafo constituye una síntesis de la obra, por resumir su experiencia de campo, aludir a las perspectivas contra las que discute e ilustrar su estilo confrontativo:

\begin{abstract}
Only one thing was clear: the archetypes of primitivist anthropology were nowhere to be found. Instead of psychosocial types like the Chief, Shaman, Warrior, and Enemy, I found diabolical spirit anthropologists, partially reconstituted souls, bulldozers, madness, addicts. Instead of animism, I found apocalypticism. Instead of jaguars who are humans, I found Indians who were animalized. Instead of wisely multinaturalist primitives crossing human/nonhuman divides at will, I found increasingly sharp and nonnegotiable divides between nature and culture, primitive and human, past and future (p. 15).
\end{abstract}

5 Ahora pasaremos a una revisión de la obra, capítulo por capítulo. En el primer capítulo, «The devil and the fetishization of tradition» («El diablo y la fetichización de la tradición »), hace una revisión del trabajo de sus predecesores y construye la figura del abujá - antropólogo en la lengua de los ayoreo -, con la que discutirá a lo largo de toda la obra.

6 Según indica Bessire, para los ayoreo el abujá es una figura trickster, lleno de dinero y dulces palabras, que busca cosas del pasado, es un ser del que desconfían y que catalogan como ayudante del demonio. Bessire analiza el uso ayoreo de la imaginería del demonio para hablar de los antropólogos. Llama la atención en el análisis la ausencia de mención a los misioneros New Tribes Mission, que han catalogado las antiguas creencias ayoreo como diabólicas (Von Bremen 1988). Bessire señala que en otros casos - como los putumayo en Colombia o los toba occidentales en Argentina - esa imaginería es usada por los indígenas para interpretar las inequidades del capitalismo y hacer frente a la explotación. Se pregunta entonces qué pueden tener en común el patrón de una mina, el administrador de una plantación y un etnógrafo de los ayoreo, y explicará que - mediante un proceso de alienación de los ayoreo y fetichización de la tradición -, así como el patrón de la mina trafica con plata o el administrador de una plantación con trabajo asalariado, un abujá trafica con tradición.

El autor analiza el trabajo de sus predecesores (Bórmida, Von Bremen, Fischermann, entre otros), y elabora una interpretación alternativa de los mitos ayoreos, que será indispensable para el argumento general de la obra. Bessire va contra el animismo y el perspectivismo que suponen que animales y humanos comparten una misma interioridad desde tiempos inmemoriales y contra la lectura de Sebag de que los animales serían humanos que renunciaron a su humanidad. Más bien, argumenta Bessire, los seres primigenios ayoreo eran proto-humanos amorales y sin sociedad. Es a través de sucesivas transformaciones que se genera la sociedad humana con una guía 
moral, ya que cada proto-humano al transformarse en animal dejaba a los demás - que aún no tenían reglas de comportamiento - una regla o enseñanza y un canto de cura. Los elementos centrales para la reproducción social no existieron siempre, sino que surgieron en esas transformaciones. Esa interpretación le permitirá al autor poner en valor la transformación, y cuestionar la atribución a los ayoreo - por parte de los abujá - de una esencia estable y una alteridad radical. Bessire analiza las consecuencias políticas que se derivan de tal violencia hermenéutica, entre ellas la negación a los ayoreo de su capacidad de transformación y auto-objetivación. Esa antropología focalizada en la tradición, argumenta el autor, implicó la omisión voluntaria de las condiciones de generación del conocimiento antropológico y de las desiguales relaciones con los ayoreo que este implicaba.

8 En el segundo capítulo, "The lost center of the world» («El centro del mundo perdido»), Bessire se adentra en la etnohistoria de los ayoreo con el fin de poner a revisión el lugar común de que los ayoreo son un pueblo guerrero, violento y territorial.

El capítulo se concentra en Echoi, salinas que eran el centro del territorio ayoreo, cerca de la frontera entre Paraguay y Bolivia, siempre descrito como escenario de numerosas batallas. Bessire encuentra relatos de batallas allí, pero también otros que sorprendentemente para lo que se conoce en la literatura - describen Echoi como un centro de alta efervescencia social, donde los grupos realizaban intercambios, arreglaban matrimonios y celebraban el importante ritual del cambio de estaciones. Algunos ayoreo lo describían como un paraíso, otros - con el recuerdo fresco de enfrentamientos-, preferían no hablar. El autor realiza entonces un recorrido etnohistórico sobre esa zona desde las misiones jesuíticas en el siglo XVIII hasta después de la Guerra del Chaco, y explica que así como varió la percepción sobre esa zona desde un paraje edénico hasta un infierno verde -, también se transformó la visión sobre los grupos que la habitaban.

El autor describe la presión constante sobre el territorio ayoreo desde comienzos del siglo xx, con el establecimiento de colonias menonitas y la Guerra del Chaco, y sugiere que la violencia externa sobre los ayoreo fue amplificada en guerras internas. Indica que hacia 1930 la paz en Echoi fue rota, hubo grandes enfrentamientos internos y el otrora lugar de encuentros se contaminó de sangre derramada. En ese punto hubiera sido interesante que el autor dialogara en mayor profundidad con la obra de la etnohistoriadora del Chaco Isabelle Combès (a la que refiere en otros puntos), quien otorga mayor peso a los factores internos, tales como la formación de la confederación del líder Uejai en los años 1920, que unió los grupos del sur contra los del norte y contra los totobiegosode (2009, p.115). En este capítulo, como a lo largo de toda la obra, merece destacarse la gran base de información de relatos orales y el análisis minucioso de informaciones en apariencia contradictorias.

11 En el tercer capítulo, «Hunting Indians » («Cazando indios»), Bessire abordará los primeros pasos de los misioneros New Tribes Mission, la noción de etnocidio, la «recolección de almas» y los antropólogos que recolectan tradición. El capítulo comienza recapitulando los eventos de fines de 1986. Un grupo de ayoreo de la misión de Campo Loro fue a contactar ayoreo totobiegosode que vivían en el monte y cuyo campamento había sido divisado por un piloto de los New Tribes Mission. Los totobiegosode, al ver llegar a los visitantes, mataron cinco de ellos antes de acordar salir en términos pacíficos. El caso tuvo revuelo internacional: varias ONG y 
agrupaciones indigenistas elaboraron documentos que hablaban de etnocidio, y condenaban la acción de los New Tribes Mission llamándola « caza de indios ». Bessire se distancia de esas interpretaciones ("This narrative is also an origin myth for the political anthropology of indigeneity", p. 86). El autor asegura que los totobiegosode no lo veían como una caza de indios - más bien estaban de acuerdo con la versión de los misioneros e inclusive estaban contentos de haber sido contactados, querían salir del monte: «We were happy when they captured us, because we no longer were happy in the forest [...]» (p. 90). Ese punto de vista era común entre los totobiegosode, afirma el autor. Para comprender el deseo de buscar parientes en el monte y traerlos al mundo de los contactados, Bessire retraza la historia de los primeros contactos en Bolivia con los New Tribes Mission. Explicará que los misioneros que recolectan almas se parecen a los antropólogos que recolectan tradición: ambos en algún punto buscan extraer algo inmaterial y extremadamente valioso - almas para unos, tradición para otros - de unos seres degradados (pecaminosos o aculturados). En la tensa situación de contacto, los ayoreo ofrecían a los misioneros una imagen aún más salvaje - por ejemplo, se incrementaba el número de infanticidios -, lo que justificaba aún más la necesidad de ir a contactarlos y salvarlos. Esa disfuncionalidad - la de que en algún punto, los misioneros creaban los salvajes que salvarían luego - era funcional a la misión. Esta dinámica contradictoria de la «caza de indios", indica el autor, sería parte también del proceso civilizatorio en general - por ejemplo con los aché en Paraguay - y hasta de la etnografía - con el caso de Jean Vellard que cazaría indios para obtener muestras de sangre. Ese no-sistema, señala Bessire, funcionaba gracias a sus contradicciones, y uno de sus pilares es justamente la noción de etnocidio ya que implica la defensa de una alteridad radical (a su vez funcional al proyecto colonial):

It does not question Indian alterity but reifies it anew. That is, the notion of ethnocide is a key colonial metanarrative that inverts and naturalizes the operations of colonial subjugation (p. 107).

12 En el capítulo 4, "Mediating the new human» ("Mediando el nuevo humano »), el autor se consagra al estudio del uso de la radio UHF en las comunidades ayoreo y a la transformación del principio anímico ayipie en el seno del nuevo contexto, Cojñone-gari (el presente en contacto con los blancos). El intenso uso de la radio en las comunidades ayoreo ha sido visto por otros investigadores como una continuidad con el pasado y como un ejercicio contrahegemónico. Es interesante en ese sentido la perspectiva diferente de Bessire, quien aborda la radio como una práctica que tiene sentido dentro del proceso - para los ayoreo - de volverse « gente nueva ». No se trata de analizar el contenido de los mensajes - que suele tratar sobre Dupade (dios) y sobre el estado de salud de parientes y amigos -, señala Bessire, sino de que la materia del principio anímico ayipie circula por las ondas de radio. El ayipie "was this kind of soul matter that Ayoreo believers imagined to have been transformed by conversion to Christianity. It was a barometer of moral standing and physical health and personal agency » (p. 116). Hay más bien una ruptura a nivel moral entre el Erami (el mundo del monte, del pasado) y el Cojñonegari (el presente en interacción con los blancos). En ese proceso hay una transformación del principio anímico ayipie, necesaria para devenir humanos nuevos. La radio es un medio para la transmisión y transformación del ayipie.

En el capítulo 5, «Apocalypse and the limits of transformation» («Apocalipsis y los límites de la transformación»), Bessire discute sobre la transformación como una estrategia propiamente ayorea de auto-objetivación, describe la cristiandad ayorea y 
explica el surgimiento de una visión apocalíptica, que permitiría a los ayoreo sobrellevar las extremas condiciones de pobreza.

El autor realiza una crítica a la visión de la conversión ayorea por parte de los antropólogos del Chaco. Estos últimos verían la conversión como una evidencia de etnocidio, o bien como una pátina que ocultaría una alteridad radical. En esos modelos, señala Bessire, la « cristiandad indígena » sólo puede ser entendida en una relación de continuidad o ruptura con el pasado, y se los ve como regímenes de valores incompatibles. El autor señala que esos modelos no sirven para comprender las practicas ayoreas de la fe, cuya heterodoxia creativa les ha servido como herramienta para volver habitable su posición como pueblos indígenas.

Los ayoreo, nos explica el autor, ven una ruptura entre la vida en el monte y la vida actual, pero no como la imaginan las ONG o los misioneros. Los ayoreo ven una discontinuidad entre la vida en el monte y la vida entre los blancos análoga a la discontinuidad entre proto-humanidad y sociedad humana en los mitos (siguiendo la interpretación que Bessire había ofrecido en el primer capítulo). Bessire explica la conversión de los ayoreo al cristianismo con la dinámica del «tocar », proceso por medio del cual se adoptaban (o capturaban) enemigos. Así, los primeros ayoreo de Bolivia al ser contactados, fueron « tocados " por los misioneros. Explica a continuación como la conversión implica una transformación - o directamente un reemplazo - del principio anímico ayipie, proceso necesario para vivir en el mundo actual.

En ese proceso de transformación, la vergüenza es el sentimiento que define el devenir "gente nueva", lo que es tratado en el capítulo 6, "Shame and the limits of the subject » («Vergüenza y los límites del sujeto »). En el dominio del monte era necesario imponerse y dominar animales, plantas y enemigos. En el nuevo dominio de Cojñonegari, la ecología moral ha cambiado y la vergüenza es parte de ella (lo que se debe en parte a la relación con Dupade, pero también al desprecio que suelen ejercer los blancos con los ayoreo).

En el capítulo 7, «Afliction and the limits of becoming» («Aflicción y los límites del devenir ») se dedica a dos casos extremos dentro del espacio ayoreo, el urusoi y las puyedie, para luego discutir las contradicciones de las políticas multiculturales en América Latina. El urusoi corresponde a una enfermedad que en épocas de pre contacto implicaba para el que la sufría volverse animal: el urusoi huía al monte y se movía y comía como una iguana. El urusoi en la era post-contacto en cambio, argumenta Bessire, se vuelve un ayoreo no contactado (rompe artilugios de los blancos). Las puyedie son las consumidoras de pasta base en Santa Cruz de la Sierra, marginales entre los marginales. Ambos comportamientos marcan los extremos del espacio moral ayoreo, "the interruption of moral humanity» (p. 175). Desde el punto de vista de los que lo padecen el urusoi y las puyedie -, es un modo radical de transformarse negativamente (p. 176). Bessire contrasta estos ejemplos de marginalidad extrema con las esperadas ventajas de una ciudadanía «post-multicultural» en América Latina. El autor analiza las contradicciones de las políticas multiculturales, entre ellas cómo se han reforzado regímenes de lo que Didier Fassin denomina «biolegitimidad» (p. 177), que produce nuevas desigualdades. Tanto es así que la cultura como derecho colectivo se basa en una noción estable y marcada de alteridad, lo que llevará a definir ciertos sujetos como « indígenas autorizados », mientras que otros «ex-primitivos »-o «aculturados »quedarán aún más marginados o, para usar la terminología del autor, « hipermarginalizados »: 
Hypermarginality can be defined as a novel regime of social depersonalization and structural violence deriving from the instrumental conflation of politically authorized culture and Indigenous biolegitimacy across distinct political domains (p. 184). analiza todo lo que se despliega alrededor de la protección de los grupos aislados: aspectos legales, la biolegitimidad, el accionar de las ONG compitiendo por financiamiento, y el relegamiento de los ya contactados que carecerían de suficiente alteridad. En este interesante capítulo Bessire analiza y desmantela los presupuestos implicados en las acciones de las ONG y explica cómo, en un intento por proteger a los indígenas aislados, terminan perjudicando a los contactados desde hace tiempo y reproduciendo lógicas coloniales. En cierto punto - explica el autor - la defensa del derecho al aislamiento se basa en la idea de que los límites de la cultura son fronteras permanentes a ser defendidas (p. 206). Esta política, con su énfasis en la estabilidad de la alteridad - una estabilidad frágil, por cierto - no es apta para pensar la transformación y la auto-representación, proceso sobre el que el autor se ha concentrado a lo largo de la obra. Hace un minucioso análisis del trabajo de las ONG en Paraguay - fundamentalmente dos que trabajan con ayoreo- y de cómo esas organizaciones no dejan protagonismo ni autonomía a los ayoreo:

Whereas Indigenous groups elsewhere have "turned to cultural forms of political struggle in direct defense of the reproduction of their lives, " both Paraguayan NGOs acted as if preserving pure culture required denying Indigenous peoples the capacity for self-objectification (p. 210).

Behold the black caiman es una obra que por su estilo y desarrollo argumental interpela al lector y lo moviliza, lo que merece destacarse de un libro. Si bien la argumentación a lo largo de la obra es clara, su discusión con ciertas propuestas teóricas no siempre lo es. A veces es difícil seguir el hilo que va de la crítica de un concepto antropológico a sus nefastas consecuencias políticas, e inclusive en ocasiones el autor llega a esquematizar en exceso el perspectivismo con el que discute (aunque también es cierto que desarrolla con mayor extensión esas críticas en otra publicación, cf. Bessire y Bond 2014). En otras ocasiones es difícil entender con precisión el uso que el autor hace de «ontología ». Hubiera sido interesante, en su crítica a la atribución de ontologías estables, que desarrollara esa noción (al respecto, ver las interesantes reflexiones sobre el uso de « ontología » en antropología en Severi 2014), ya que de hecho es una noción útil para el autor (quien hablará de "ontological self-determination", "ontological transformation", "ontological senses of being in the world", "ontological murkiness of the colonial encounter ", etc.). La obra presenta, no obstante, un claro abordaje en pos del estudio del devenir ayoreo.

Behold the black caiman. A chronicle of Ayoreo life es una obra ante la cual es difícil permanecer indiferente - sobre todo si uno es antropólogo o miembro de una ONG dado que los interrogantes que despierta sobre el papel de la disciplina y las políticas indigenistas trascienden ampliamente el caso ayoreo. Tanto por su sólida base etnográfica, como por su actualidad y relevancia, Behold the black caiman es una obra de referencia en la etnografía del Chaco. 
BIBLIOGRAFÍA

BESSIRE Lucas y David BOND

2014, « Ontological anthropology and the deferral of critique », in American ethnologist, 41 (3), p. $440-456$.

BREMEN Volker von

1988, Los ayoreos cazados, Servicios Profesionales Socio-Antropológicos y Jurídicos, Asunción.

coMBÈs Isabelle

2009, Zamucos, Éd. Nómades/Insituto de Misionología (Scripta autochtona, 1), Cochabamba.

SEVERI Carlo

2014, « Transmutating beings. A proposal for an anthropology of thought », in HAU: Journal of ethnographic theory, 4 (2), p. 41-71.

\section{AUTORES}

\section{ALFONSO OTAEGUI}

Instituto de Ciencias Antropológicas, Facultad de Filosofía y Letras, Universidad de Buenos Aires 